\title{
A software simulator for the Herschel-SPIRE imaging photometer
}

\author{
Bruce Sibthorpe ${ }^{*}$, Adam Woodcraft, Matthew Griffin, Steven Lloyd Watkin \\ Department of Physics \& Astronomy, Cardiff University, Cardiff CF24 3YB, Wales, UK
}

\begin{abstract}
SPIRE, the Spectral and Photometric Imaging Receiver, is one of three instruments to be flown on ESA's Herschel Space Observatory. It contains a three-band submillimetre camera and an imaging Fourier transform spectrometer, and uses arrays of feedhorn-coupled bolometric detectors operating at a temperature of $300 \mathrm{mK}$. Detailed software simulators are being developed for the SPIRE photometer and spectrometer. The photometer simulator is based on an adaptable modular representation of the relevant instrument and telescope subsystems, and is designed to produce highly realistic science and housekeeping data timelines. It will be used for a variety of purposes, including instrument characterisation during ground testing and in orbit, testing and optimisation of operating modes and strategies, evaluation of data reduction software using simulated data streams (derived by "observing" a simulated sky intensity distribution with the simulator), observing time estimation, and diagnostics of instrument systematics. In this paper we present the current status of the photometer simulator and the future development and implementation strategy.
\end{abstract}

Keywords: Herschel, SPIRE, Simulator, Photometer

\section{INTRODUCTION}

SPIRE $^{1}$, the Spectral and Photometric Imaging Receiver, is one of three instruments to be flown on ESA's Herschel Space Observatory ${ }^{2}$, due for launch in 2007. It covers the submillimetre band between 200 and $670 \mu \mathrm{m}$, and comprises a three-band imaging photometer and an imaging Fourier transform spectrometer (FTS). The main scientific objectives for SPIRE are deep extragalactic and galactic imaging surveys and spectroscopy of star-forming regions in our own and nearby galaxies. The instrument and the Herschel telescope and cryostat form a complex system, and the manner in which this system determines the science and housekeeping data must be well understood in order to make sure that the instrument is operated most effectively. It is for this reason we have begun development of detailed simulators for the SPIRE photometer and FTS. The photometer simulator is described in this paper, and the status of the FTS simulator is described by Linder et al. ${ }^{3}$

\subsection{THE SPIRE PHOTOMETER}

The SPIRE three-band photometer has a field of view of $4 \times 8$ arcminutes, observed simultaneously in spectral bands centred approximately at 250,360 and $520 \mu \mathrm{m}$, made possible by the use of dichroic beam dividers separating out the three bands. The angular resolution is determined by the telescope diffraction limit, with FWHM beam widths of approximately 18, 25 and 36 arcseconds at 250, 360 and $520 \mu \mathrm{m}$ respectively. An internal beam steering mirror ${ }^{4}$ (BSM) can be used for spatial modulation of the telescope beam, and observations can also be made by scanning the telescope without chopping.

The SPIRE photometer layout, illustrated in Figure 1, is an all-reflective system except for two dichroic beam dividers used to direct the three wavelength bands onto the bolometer arrays ${ }^{5}$, and various edge filters to reject out-of-band radiation. The optical design gives diffraction-limited imaging across a 4 × 8 arcminute field of view, simultaneously observed by all three detector arrays. The detector bodies and dichroics are supported from an enclosure maintained at a temperature of approximately $2 \mathrm{~K}$, with the rest of the photometer elements mounted to an optical bench panel at $4.5 \mathrm{~K}$. The detector arrays, feedhorns, and the final filters are thermally isolated from the $2 \mathrm{~K}$ structure by Kevlar cords, and

\footnotetext{
* Further author information: E-mail: Bruce.Sibthorpe@astro.cf.ac.uk; Telephone: +44-29-2087-6274; Fax: +44-29-2087-4056
} 
are cooled to approximately $300 \mathrm{mK}$ by a thermal strap to a ${ }^{3} \mathrm{He}$ refrigerator ${ }^{6}$. The photometer field of view can be steered in two axes by the BSM, which also houses a thermal source used for secondary calibration.

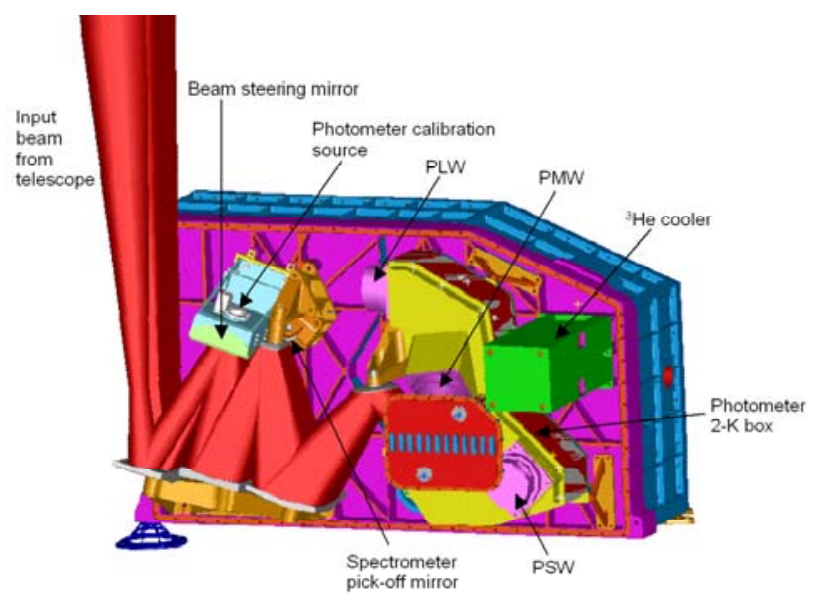

Figure 1: General view and schematic of the SPIRE photometer (covers removed). PSW, PMW and PLW denote the 250, 360, and $520 \mu \mathrm{m}$ detector arrays respectively.

The photometer input optics are shared by the spectrometer up to a pick-off mirror where the separate spectrometer field of view is directed through a hole in the optical bench panel to the other side of the instrument.

The layout of SPIRE's photometer arrays is shown schematically in Figure 2. The arrays have the same field of view on the sky but are shown separately here for clarity. There are 139, 88 and 43 detectors in the 250, 460 and $520 \mu \mathrm{m}$ bands respectively. There are several sets of three detectors for which the beams at the three wavelengths are exactly coaligned on the sky, indicated by the shaded circles in Figure 2. When chopping (by 126 arcseconds) between these sets of pixels the source is observed in all three wavebands at all times.

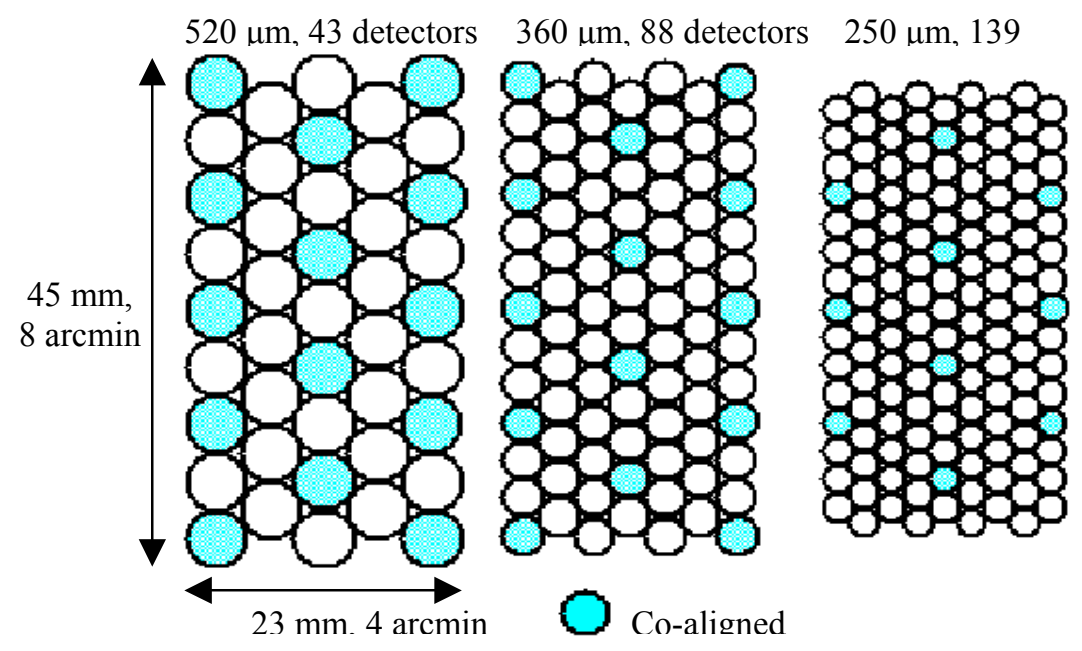

Figure 2: Layout of the three photometer arrays, including co-aligned pixels

SPIRE uses Neutron Transmutation Doped (NTD) germanium spider web bolometers, which are read out thorough lock in amplifier (LIA) circuits. Each detector has its own dedicated LIA circuit with a $5 \mathrm{~Hz}$ analogue bandpass filter. This 
filter is matched to the signal modulation frequency, thereby allowing all noise outside of this frequency band to be eliminated from the measurement. All detectors operate at a sampling rate of up to $28 \mathrm{~Hz}$ for the photometer, and $80 \mathrm{~Hz}$ for the spectrometer.

\subsection{THE PHOTOMETER SIMULATOR}

The photometer simulator is designed to be a detailed and flexible computer representation of the entire photometer system, taking into account both telescope and instrument systems, and to produce highly realistic data streams for the main observing modes, generated by "observing" and artificial sky with the simulated instrument. This will allow the operating modes and data reduction software to be tested, and instrument systematics to be diagnosed and evaluated. In addition to sampled data and housekeeping timelines, other timelines unobtainable from the physical system will be available for analysis allowing the complete system performance to be scrutinized. Data generated will be reduced using a combination of SPIRE data reduction software and routines specifically developed for the analysis of simulator output. Calculations to evaluate the overall SPIRE system sensitivity have been carried out ${ }^{1}$, however the simulator will constitute a much more sophisticated and versatile tool for evaluating the photometer performance, and how it is affected by the detailed observing mode parameters. It will also be used useful as a diagnostic tool for the instrument behaviour both in ground testing and in flight operation.

\section{PHOTOMETER OBSERVING MODES}

In order to simulate accurately the SPIRE photometer outputs it is necessary to represent all of the planned observing modes. This includes functions required to carry out instrument setup and calibration as well as scientific observations. In this section we summarize the various Photometer Observatory Functions (POFs) available for SPIRE and the basic methods by which these functions are carried out.

\subsection{OBSERVATORY FUNCTIONS}

There are nine POFs, six relating to scientific observations, and three to instrument calibration and setup as summarized in the Table 1. The six scientific observing modes can be split into one of three main categories, point source photometry, field mapping, and scan mapping.

Table 1: Summary of SPIRE photometer observing modes

\begin{tabular}{llll}
\hline \multirow{2}{*}{ Observation } & $\begin{array}{c}\text { Observatory } \\
\text { Function }\end{array}$ & Name & Comments \\
\hline \multirow{2}{*}{ Point source photometry } & POF1 & Chop without jiggling & Accurate pointing and source position \\
\cline { 2 - 4 } & POF2 & Seven-point jiggle map & In accurate pointing or source position \\
\hline \multirow{2}{*}{ Field mapping } & POF3 & Jiggle mapping & Field mapping \\
\cline { 2 - 4 } Scan mapping & POF4 & Raster map & Extended field mapping \\
\hline Peak-up & POF5 & Scan map without chopping & Large-area mapping \\
\cline { 2 - 4 } Calibrate & POF6 & Scan map with chopping & Large-area mapping \\
\hline \multirow{2}{*}{ Engineering modes } & POF7 & Photometer peak-up & Determination of pointing offsets \\
\hline
\end{tabular}

POF commands are split into two categories, Observatory Functions and Instrument Functions. Observatory functions are carried out by the telescope (primarily pointing, scanning and nodding). Instrument functions are carried out by the SPIRE instrument hardware, such as control of the BSM to produce beam chopping and the sampling of the detectors. Combinations of these two commanded timelines produce all POFs specified above. 


\subsection{POINT SOURCE PHOTOMETRY (POFS 1 \& 2)}

Point source photometry (POF 1) is used for observing isolated compact objects and is identical to the standard observing mode used on ground-based submillimetre telescopes. Observations can be made by simply chopping the source while pointing the telescope precisely and nodding if required. If telescope pointing is not sufficiently accurate however, or the source position is not well defined, then a seven-point jiggle map may be used (POF 2). This is constituted by seven individual POF 1 observations carried out in a symmetrical pattern centred on the source, with the BSM being offset by some angle $\theta$ for each point (see Figure 3 ).

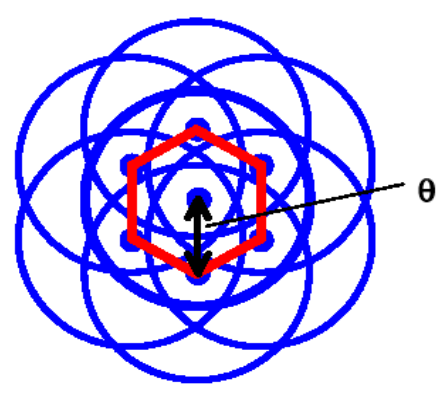

Figure 3: Seven-point hexagonal jiggle pattern.

\subsection{FIELD MAPPING}

POF 3 (field mapping) is for mapping of relatively small objects or regions, no greater than a few arcminutes in size. Implementation is identical to that of POF 1 but with a number of chop positions. In order to achieve full sampling in each waveband 64 jiggle points must be used. POF 4 field mapping carries out a POF 3 observation at each point in a raster scan and can therefore be used to scan areas larger than the SPIRE field of view.

\subsection{SCAN MAPPING}

Scan mapping (POF 5) is used in order to for large area maps - much bigger than the SPIRE field of view. In this configuration all three arrays observe simultaneously while the telescope scans across the sky at a rate of up to 1 arcminute per second. However should 1/f noise be significant, additional chopping motion can be added to introduce a higher frequency signal modulation. To achieve full spatial sampling with each of the hexagonally packed arrays the telescope scan angle must have a particular value of $14.5^{\circ}$ with respect to one of the array axes $(\mathrm{Y}$ or $\mathrm{Z})$ as shown below.

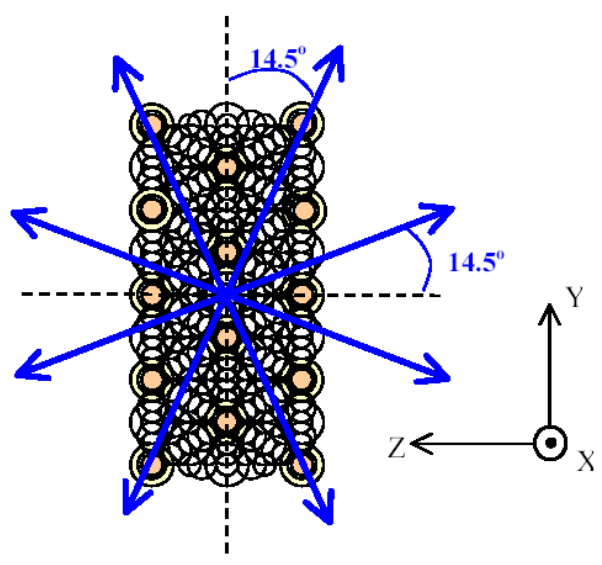

Figure 4: Fully sampled scan map angles 


\section{SIMULATOR ARCHITECTURE AND MODULAR STRUCTURE}

\subsection{ARCHITECTURE}

The simulator is a modular computer program written in Interactive Data Language (IDL). Each physical system, e.g. the detector properties and readout electronics, is represented by a separate module, governed and called when required by a 'core' program. All system parameters are written to a 'global workspace' (GW) from a list contained within each module, and are again controlled by the core program. This control includes the ownership and visibility restrictions of specific parameters contained within the GW.

Individual modules are entirely self-contained allowing for the update of any system component without effect to other parts of the simulator. This modularity is achieved by having a well-defined set of input and output parameters. Input parameters are defined by or produced by other modules. Output parameters are computed within the module and are functions of the given input. This modular architecture will allow the simulator development to proceed in an organised way, with increasingly sophisticated internal module structure being implemented without affecting any other modules. In addition to these two parameter types there also exist internal parameters, defined as a parameter set or derived, and used solely within a module.

Three different kinds of timelines are implemented. Command timelines are timelines of commanded parameters, such as telescope or BSM position, which are available without error for the data analysis. Measured timelines are timelines of sampled science or housekeeping data, simulating what will be available in the telemetry stream. Hidden timelines are produced by the module corresponding to quantities which affect the data but cannot in reality be directly available to the instrument user - an example is the actual telescope pointing timeline as opposed to the commanded timeline: the former is equivalent to the latter but with added noise the exact details of which will not be available from the data stream.

In order to be consistent throughout the simulator, all input and output parameters are passed in SI units. While it is not essential to keep to this convention within a module it is preferred unless another more natural unit is applicable. This requirement is set to preserve the modularity and reduce confusion between different users.

\subsection{MODULES}

Table 2 contains a full list of all modules intended for inclusion within the simulator once fully complete. Combined they represent all aspects involved in carrying out photometer observations with the SPIRE/Herschel system.

The simulator uses a time stepping sequence to create its timelines. Each time step is $10 \mathrm{~ms}$ and is referenced to a master clock contained within the core program, with each module being called as required each step. There are some "static" modules, whose output parameters do not change with time. These are called only once in the initialization run carried out at the beginning of the simulation. These include modules for the sky simulation and the optical properties of the system. Other modules can also be included at this point should a simplified simulation be required, e.g. the thermal system module, i.e. a simulation with no temperature fluctuations. The result is a more efficient, and thus more flexible simulator when carrying out simple simulations.

\subsection{CORE}

The primary function of the core program is to manage and carry out specified simulations efficiently. Each module communicates solely with the core, with information being transferred through the GW.

Each module is represented in the core as an IDL object. All objects are defined within a module-specific defining routine, detailing all parameters owned (primarily output parameters) and readable (input parameters) by that module. When created, module defining routines inherit the properties and setup of a module template, providing standardization of objects throughout the core. 
Table 2: Simulator module list

\begin{tabular}{|c|c|c|c|}
\hline No. & Module & Abbreviation & Description \\
\hline 0 & Sky Simulator & SKYSIM & $\begin{array}{l}\text { Produces a simulation of the area of sky to be observed, with a } \\
\text { resolution finer than the SPIRE beam. A nominal pixel size of } \\
2 \text { " has been chosen, which is much smaller than SPIRE's } \\
\text { narrowest beam }(18 " 250 \mu \mathrm{m}) \text {. }\end{array}$ \\
\hline 1 & Input & INPUT & $\begin{array}{l}\text { Specifies the observation in "astronomer's terms" - this will be } \\
\text { equivalent to the Astronomical Observation Template (AOT) } \\
\text { that will be used by observers to specify their observations. }\end{array}$ \\
\hline 2 & $\begin{array}{l}\text { Observatory } \\
\text { Function }\end{array}$ & OBSFUN & $\begin{array}{l}\text { Specifies the photometer observing node to be simulated in } \\
\text { terms of the appropriate Observatory Function and its } \\
\text { parameters, and defines the commanded telescope pointing } \\
\text { timeline. }\end{array}$ \\
\hline 3 & Optical System & OPTICS & $\begin{array}{l}\text { Represents the relevant optical properties and parameters of the } \\
\text { telescope and the SPIRE photometer (including all mirrors, } \\
\text { filters, dichroics and detector feedhorns), the beam profiles, and } \\
\text { the positional mapping of the detectors on the sky. }\end{array}$ \\
\hline 4 & Thermal System & THERMAL & $\begin{array}{l}\text { Contains all information on the temperatures of the instrument } \\
\text { and the telescope, and their temporal fluctuations. }\end{array}$ \\
\hline 5 & $\begin{array}{l}\text { Telescope Pointing } \\
\text { Timeline Generator }\end{array}$ & POINTING & $\begin{array}{l}\text { Produces the actual telescope boresight pointing timeline for the } \\
\text { period of the observation, so that each detector sample can be } \\
\text { associated with a particular point on the sky. }\end{array}$ \\
\hline 6 & Beam Steering Mirror & BSM & $\begin{array}{l}\text { Produces the BSM timeline in the form of an additional pointing } \\
\text { timeline to be superimposed on that of the telescope. }\end{array}$ \\
\hline 7 & $\begin{array}{l}\text { Incident background } \\
\text { power timeline } \\
\text { generator }\end{array}$ & BACKGROUND & $\begin{array}{l}\text { Produces a timeline for the background power incident on each } \\
\text { detector, due to all contributions from the telescope and } \\
\text { instrument, including any contribution from their thermal } \\
\text { fluctuations. }\end{array}$ \\
\hline 8 & $\begin{array}{l}\text { Astronomical power } \\
\text { timeline generator }\end{array}$ & SIGNAL & $\begin{array}{l}\text { Generates a timeline for the power on each detector from the } \\
\text { astronomical sky. }\end{array}$ \\
\hline 9 & $\begin{array}{l}\text { Bolometer voltage } \\
\text { timeline generator }\end{array}$ & DETECTORS & $\begin{array}{l}\text { Produces an output voltage timeline for each detector channel } \\
\text { based on the inputs from } 7 \text { and } 8 \text { and a physical model of the } \\
\text { detector and its analogue electronics chain. }\end{array}$ \\
\hline 10 & $\begin{array}{c}\text { Science Data } \\
\text { Timeline Generator }\end{array}$ & DATA & $\begin{array}{l}\text { Based on the output from 9, produces digitised timelines for } \\
\text { each detector channel. }\end{array}$ \\
\hline 11 & $\begin{array}{l}\text { Housekeeping Data } \\
\text { Timeline Generator }\end{array}$ & $\mathrm{HK}$ & Produces timelines for all instrument temperatures \\
\hline 12 & $\begin{array}{l}\text { Calibrator Power } \\
\text { Timeline Generator }\end{array}$ & PCAL & $\begin{array}{l}\text { Produces a timeline of the power incident on each detector from } \\
\text { the photometer internal calibration source if it is being operated }\end{array}$ \\
\hline
\end{tabular}

In addition to the parameter setup and read restrictions, the module defining routine also incorporates three 'process command' subroutines. The first is an initialization process, called by the core following setup of the module objects. Its purpose is to set up each module for the following simulation. In the case of 'static' modules however, the entire module may be called, run, and its output logged solely in this initialization phase. Following this the two step commands are called. These routines compute the output of a module at a given time step and are called when each a time step is taken. The simulator uses both of step command modes every time a step is taken.

The first mode calls all modules and computes their outputs based on the parameter values set in the GW after the previous step. The GW is then updated to include all newly computed data. Consequently the order in which the module are called to carry out a step is not important in this mode. 
Some operations carried out by the simulator however take place on timescales much shorter than that of the simulator time step. As a result, there exists the situation in which the first mode is not suitable, as a parameter needed by one module may only be calculated within the same time step as it is required, and thus not be available as an input. A second mode accounts for this situation by updating the GW upon completion of each module call. In this mode the module ordering is extremely important in order to ensure the correct flow of information through the system (see Figure 5).

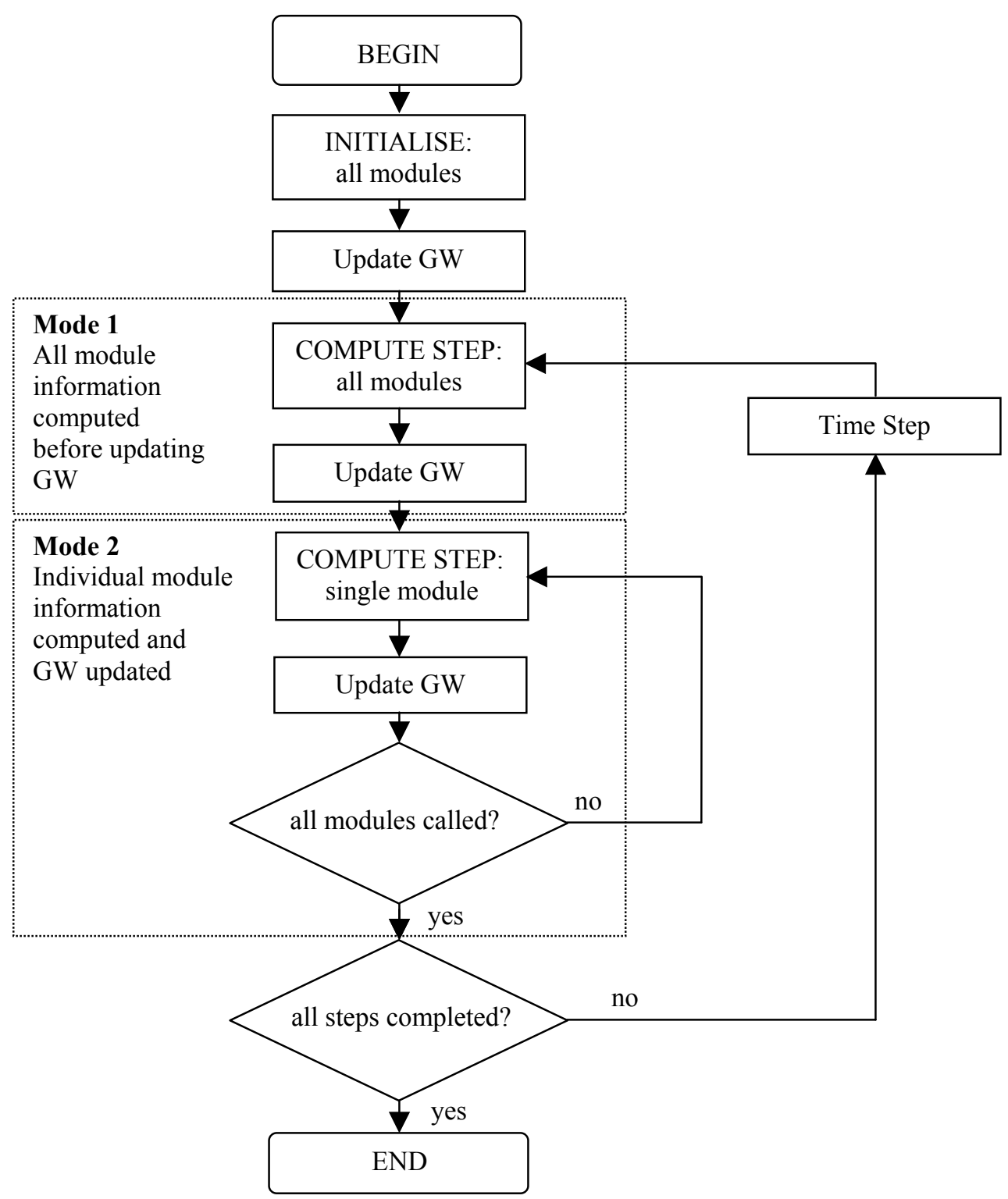

Figure 5: Simulator time step command flow-chart 


\section{SIMULATOR DEVELOPMENT STATUS (SPS V1.0)}

Implementation of the simulator is still in the early stages. All modules have been fully specified in terms of input and output parameters, and a simplified version of the complete system with a cut down list of modules has been developed and tested. The primary aspects of modules 0,2 (POFs 1 and 5 only), 3, 5, 6, 7, 8 and 9 are operational. At present only one detector is represented in each waveband. Noise contributions are not implemented separately in each internal timeline, but can be added at the system output in the form of a white or $1 / \mathrm{f}$ noise addition to the bolometer signal timelines. The careful construction in both current module design and core program architecture will allow for a simple replication to full detector arrays and increased fidelity within the modules.

\section{PRELIMINARY RESULTS}

In the course of simulator development and testing to date, the effect of $1 / \mathrm{f}$ noise on a chopped observation was investigated. Simulated chopped observations (POF1) were carried out using a simple simulated 2-D sky containing a single delta function source. Four thousand chop cycles were performed at $2 \mathrm{~Hz}$ frequency with a detector sampling rate of $32 \mathrm{~Hz}$, giving a total scan time of $2000 \mathrm{~s}$. Following this a 1/f noise timeline was generated by taking the Fourier transform of a white noise data timeline in the time domain, and obtaining the white noise power spectrum. A 1/f spectrum was then imposed on the white noise power spectrum and the inverse transform carried out (see Figure 6). The signal and $1 / \mathrm{f}$ noise timelines were then summed to produce a simulated observation data timeline.

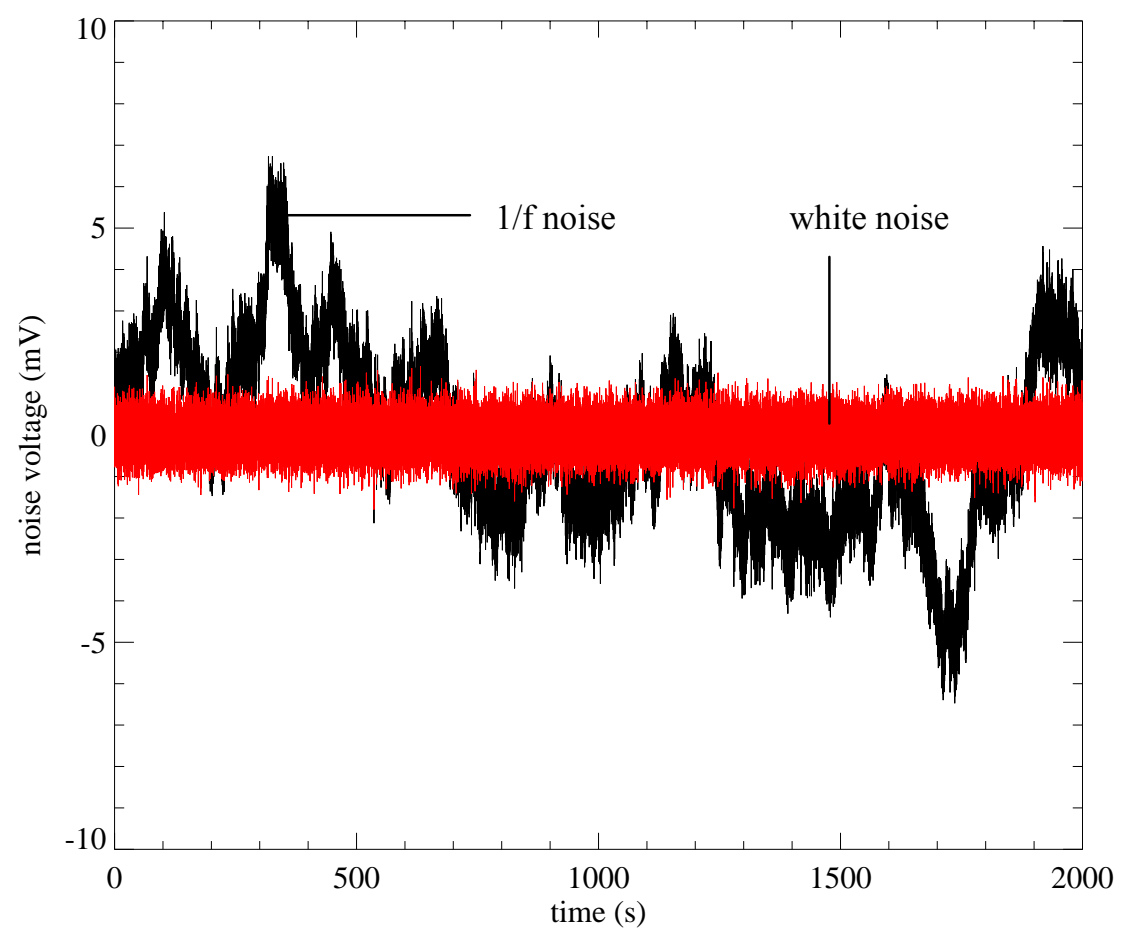

Figure 6: Simulated white noise and derived 1/f noise with a knee frequency of $0.5 \mathrm{~Hz}$ added to bolometer voltage timeline output 


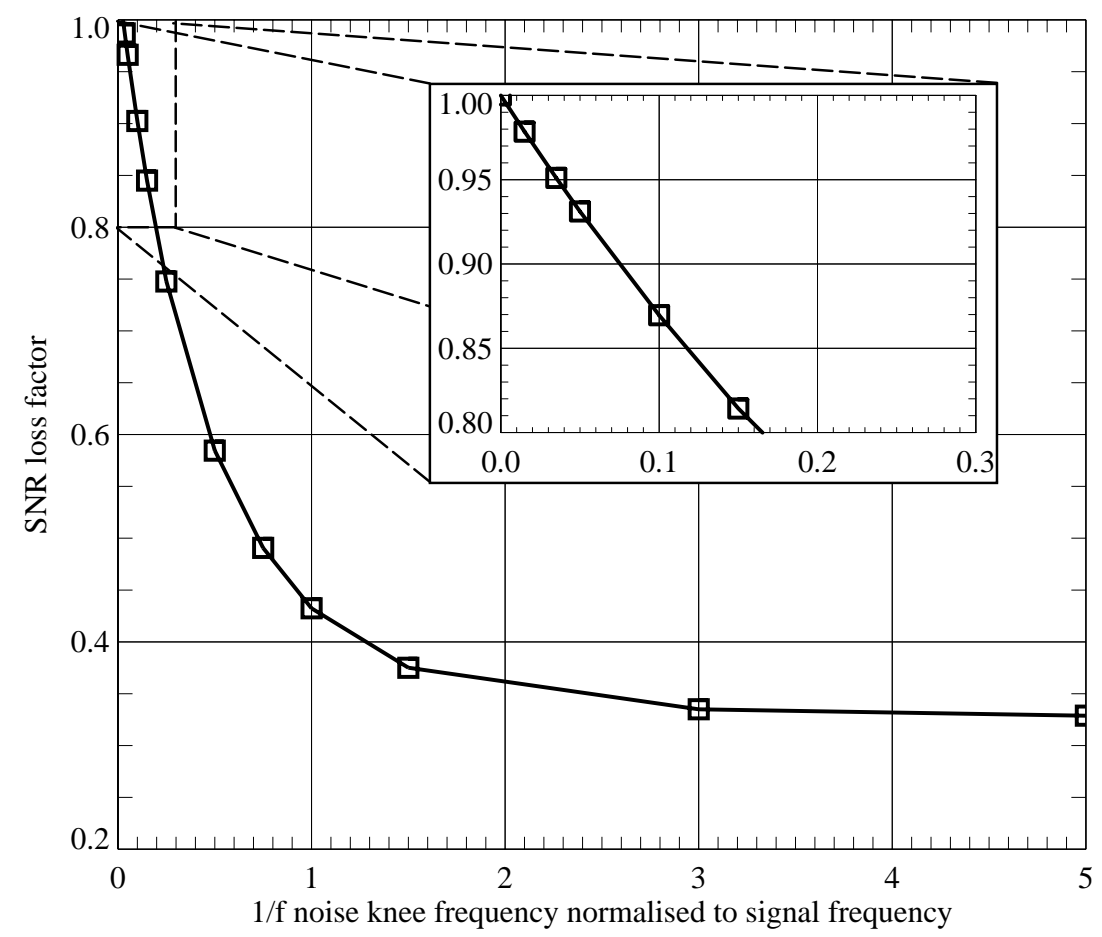

Figure 7: SNR loss as a function of increasing 1/f noise knee frequency for chopped observation

Some results from these early tests can be seen in Figure 7, showing a strong decrease in signal to noise ratio (SNR) with increasing 1/f knee frequency, with the SNR being half its value for pure white noise at a knee frequency of approximately $0.6 \mathrm{~Hz}$. Adopting the criterion that the SNR loss factor $>0.9$, requires a $1 / \mathrm{f}$ knee frequency less than one tenth of the signal frequency.

\section{FUTURE PLANS}

Future plans for the simulator include further development of the system to incorporate additional noise contributions (e.g. pointing and thermal fluctuation noise), full detector array representation, simulation of all observing modes, and the investigation of the impact of observing parameters and noise characteristics on SNR and data quality. These will require additional data analysis routines to be written and an enlargement of the simulator team as the project develops.

\section{ACKNOWLEDGEMENTS}

The authors acknowledge the UK Particle Physics and Astronomy Research Council for financial support.

\section{REFERENCES}

1. M.J. Griffin, B. Swinyard, and L. Vigroux, The Herschel-SPIRE instrument, Proc. SPIE 5847 (this volume), Glasgow 21-25 June 2004. 
2. G. Pilbratt, Herschel Mission: status and observing opportunities, Proc. SPIE 5487 (this volume), Glasgow, 21-25 June 2004.

3. J. Lindner, D. Naylor, B. Swinyard, Simulation of the Performance of the ESA's Herschel/SPIRE Imaging Fourier Transform Spectrometer, Proc. SPIE 5847 (this volume), Glasgow 21-25 June 2004.

4. I. Pain, G. S. Wright, B. Stobie, SPIRE beam steering mirror: a cryogenic 2 axis mechanism for the Herschel Space Observatory, Proc. SPIE 4850, 619-627, 2002.

5. A. D. Turner, J. J. Bock, J. W. Beeman, J. Glenn, P. C. Hargrave, V. V. Hristov, H. T. Nguyen, F. Rahman, S. Sethuraman, A. L. Woodcraft, $\mathrm{Si}_{3} \mathrm{~N}_{4}$ micromesh bolometer array for submillimeter astrophysics, Appl. Opt. Vol. 40, No. 28, 2001 4921-4932

6. L. Duband, Proc. of ESA symposium on The Far Infrared and Submillimetre Universe, Grenoble, 15-17 April 1997, ESA SP-401, 357, 1997. 